\title{
Biocompatible combinations of nisin and licorice polyphenols exert synergistic bactericidal effects against Enterococcus faecalis and inhibit NF-KB activation in monocytes
}

\author{
Daniel Grenier * (10, Eve Marcoux, Jabrane Azelmat, Amel Ben Lagha and Philippe Gauthier
}

\begin{abstract}
Enterococcus faecalis is one of the bacterial species most frequently isolated from persistent endodontic and apical periodontal infections. The aim of the present study was to evaluate the synergistic antibacterial effects of nisin and selected licorice polyphenols (glabridin, licoricidin, licochalcone A) against planktonic and biofilm-embedded E. faecalis cells. The biocompatibility and anti-inflammatory properties of the nisin/licorice polyphenol combinations were also investigated. The lantibiotic bacteriocin (nisin), the two isoflavonoids (glabridin, licoricidin), and the chalcone (licochalcone A) efficiently inhibited the growth of E. faecalis, with MICs ranging from 6.25 to $25 \mu \mathrm{g} / \mathrm{mL}$. Combining nisin with each licorice polyphenol individually resulted in a significant synergistic antibacterial effect. Following a 30-min contact, nisin in combination with either glabridin, licoricidin, or licochalcone A caused significant biofilm killing. The nisin/licorice polyphenol combinations had no cytotoxic effects (oral epithelial cells, gingival fibroblasts, and stem cells of the apical papilla), with the exception of nisin/glabridin, when used at their MICs. Lastly, we showed that nisin/glabridin, nisin/licoricidin, and nisin/licochalcone A inhibit NF-kB activation induced by E. faecalis in a monocyte model, suggesting that these combinations possess anti-inflammatory properties. The present study provides evidence that combinations of nisin and glabridin, licoricidin, or licochalcone A show promise as root canal disinfection agents.
\end{abstract}

Keywords: Enterococcus faecalis, Nisin, Licorice, Polyphenols, Biofilm, Synergy, NF-kB, Monocytes

\section{Key points}

Nisin and licorice polyphenols exert synergistic antibacterial activity.

Nisin and licorice polyphenols did not show any cytotoxicity on different oral cell types.

Combinations of nisin and licorice polyphenols possess anti-inflammatory properties.
*Correspondence: Daniel.Grenier@greb.ulaval.ca Oral Ecology Research Group, Faculty of Dentistry, Université Laval, 2420 de la Terrasse, Quebec City, QC G1V OA6, Canada

\section{Introduction}

Although many microorganisms colonizing the oral cavity can penetrate and infect the dental pulp, only a limited number of species are truly capable of doing so given the particular environment of the root canal (Siqueira and Rocas 2009). Once they gain access to the complex root canal system, bacteria adhere to the dentine surface and form a biofilm, which is a complex aggregation of microorganisms entrapped in a polymer network mainly composed of polysaccharides and DNA. Enterococcus faecalis is one of the bacterial species most frequently isolated from persistent endodontic and apical periodontal infections (Roças et al. 2004; Sedgley et al. 2006). The exact source of this 
Gram-positive facultative anaerobic bacterium is not clear, although there is evidence that E. faecalis may have an exogenous origin rather than being a permanent resident of the oral cavity (Vidana et al. 2011). E. faecalis has the ability to form a dense biofilm, which makes this species much more resistant to host immune defenses as well as to antimicrobial agents used for root canal disinfection (Duggan and Sedgley 2007).

An important aspect of endodontic therapy is the use of irrigating solutions possessing antimicrobial activity against both planktonic and biofilm-embedded microorganisms. Endodontic treatments are aimed at eliminating the microorganisms in the root canal. However, given the anatomic complexity of the root canal system, this can be a challenge. If the endodontic treatment fails, the residual bacteria and their toxins may affect the periradicular tissues and create an inflammatory response resulting in apical periodontitis, which is characterized by the recruitment of inflammatory cells, including polymorphonuclear neutrophils and monocytes (Gomes and Herrera 2018). When the NF-kB signaling pathway is activated by bacteria or their cell surface components such as lipopolysaccharide (LPS) and lipoteichoic acid, these cells release pro-inflammatory mediators that contribute to tissue destruction (Gomes and Herrera 2018).

Sodium hypochlorite is one of the most commonly used root canal irrigating agents as it can dissolve organic matter and is a strong disinfectant (Goncalves et al. 2016). However, the extrusion of sodium hypochlorite beyond the apical foramen or through a perforation can lead to complications, from minor to severe tissue damage (De Sermeno et al. 2009). Because of this, research aimed at identifying novel non-cytotoxic irrigating agents with broad-spectrum antimicrobial activity is of great interest. Antimicrobial peptides and plant polyphenols may be promising candidates in this regard.

Nisin is a broad-spectrum bacteriocin naturally produced by Lactococcus lactis that has been extensively studied in the food industry (Hansen 1994). This antimicrobial peptide (34 amino acid residues) is currently approved for use as a food preservative in 48 countries. Nisin is particularly effective in killing Gram-positive bacteria by interacting with their negatively charged membranes, leading to pore formation (Lubelski et al. 2008). Polyphenols are plant secondary metabolites and are classified into different families based on their chemical structures (Fraga et al. 2019). Previous investigations have shown that several classes of polyphenols exhibit a broad spectrum of antimicrobial activity (Daglia 2012). More specifically, licorice-derived chalcones and isoflavonoids have been reported to possess marked antibacterial activity against both Gram-positive and Gram-negative bacteria (Messier et al. 2012).
The aim of the present study was to evaluate the synergistic antibacterial effects of nisin and selected licorice polyphenols (glabridin, licoricidin, licochalcone A) against planktonic and biofilm-embedded E. faecalis cells. The biocompatibility and anti-inflammatory properties of the nisin/licorice polyphenol combinations were also investigated.

\section{Materials and methods Compounds}

Glabridin (Wako Chemicals, Richmond, VA, USA) and licochalcone A (Enzo Life Sciences, Inc., Farmingdale, NY, USA) were prepared in $95 \%(\mathrm{v} / \mathrm{v})$ ethanol, while licoricidin (EMMX Biotechnology, Lake Forest, CA, USA) was prepared in dimethyl sulfoxide. Stock solutions $(20 \mathrm{mg} / \mathrm{mL})$ were kept in the dark at $4{ }^{\circ} \mathrm{C}$ for up to 1 month. Given that these stock solutions were highly diluted in the assays described below, neither ethanol nor dimethyl sulfoxide caused any biological effects (data not shown). Nisin A (Sigma-Aldrich Canada Co., Oakville, $\mathrm{ON}$, Canada) was prepared in $0.02 \mathrm{~N} \mathrm{HCl}$ at a concentration of $750 \mu \mathrm{g} / \mathrm{mL}$ and was sterilized by filtration through a $0.22-\mu \mathrm{m}$ pore size membrane filter. Chlorhexidine digluconate (Sigma-Aldrich Canada Co.,), which was used as a positive antimicrobial control, was prepared in distilled water at a concentration of $5 \mathrm{mg} / \mathrm{mL}$ and was sterilized by filtration through a $0.22-\mu \mathrm{m}$ pore size membrane filter.

\section{Bacteria and growth conditions}

The reference strain E. faecalis ATCC 19433 as well as two clinical isolates of E. faecalis (0DOT, and 1DOT) were used. They were grown in Brain Heart Infusion broth (BHI; BBL Microbiology Systems, Mississauga, ON, Canada) supplemented with $0.5 \%$ glucose at $37^{\circ} \mathrm{C}$ in an anaerobic chamber $\left(80 \% \mathrm{~N}_{2} / 10 \% \mathrm{H}_{2} / 10 \% \mathrm{CO}_{2}\right)$.

\section{Determination of minimum inhibitory and minimum bactericidal concentrations}

The minimum inhibitory concentration (MIC) and minimum bactericidal concentration (MBC) values of nisin and the licorice polyphenols were assessed using the microdilution method as described by the Clinical and Laboratory Standards Institute (Wayne 2012). The bacterial suspension and two-fold serial dilutions of the compounds (from $200 \mu \mathrm{g} / \mathrm{mL}$ ) diluted in culture medium were added to the wells of a 96-well microplate. Wells with no compounds (only carrier solvent) were used as controls (100\% growth). After a 24-h incubation, microbial growth was assessed by recording the optical density at $660 \mathrm{~nm}\left(\mathrm{OD}_{660}\right)$ using a Synergy 2 microplate reader (BioTek Instruments, Winooski, VT, USA). The MIC values are the lowest concentrations of the compounds that 
completely inhibited bacterial growth. To determine the MBC values, $5-\mu \mathrm{L}$ aliquots from the wells with no visible growth were spread on BHI agar plates, which were incubated for 3 days at $37^{\circ} \mathrm{C}$. The MBC values are the lowest concentrations at which no colony formation occurred. Assays were performed in triplicate in two independent experiments to ensure reproducibility. A representative set of data is presented.

\section{Biofilm formation}

E. faecalis ATCC 19433 was grown $(24 \mathrm{~h})$ in the wells of a flat-bottomed 96-well microplate as described above in the absence (control) and presence of either nisin or licorice polyphenols at sub-inhibitory concentrations corresponding to $1 / 4$ MIC. The medium, free-floating bacteria, and loosely-bound biofilm were then removed by aspiration, and the wells were gently washed three times with $50 \mathrm{mM}$ phosphate-buffered saline $(\mathrm{pH} 7.2$; PBS). The biofilms were stained with $0.04 \%$ crystal violet $(100 \mu \mathrm{L})$ for $10 \mathrm{~min}$. The wells were washed three times with PBS to remove unbound crystal violet dye and were dried for $2 \mathrm{~h}$ at $37^{\circ} \mathrm{C}$. After adding $100 \mu \mathrm{L}$ of $95 \%(\mathrm{v} / \mathrm{v})$ ethanol to each well, the microplate was shaken for $10 \mathrm{~min}$ to release the dye from the biofilms, and the absorbance at $550 \mathrm{~nm}\left(\mathrm{~A}_{550}\right)$ was recorded using a Synergy 2 microplate reader. Assays were performed in triplicate in two independent experiments, and the means \pm standard deviations were calculated.

\section{Synergistic antibacterial interactions}

The synergistic antibacterial effects against E. faecalis ATCC 19433 of nisin in combination with the licorice polyphenols were evaluated using the checkerboard method (Eliopoulos and Moellering 1996). Compound A was serially diluted in culture medium $(100 \mu \mathrm{L})$ along the ordinate of a 96-well microplate, while compound B was serially diluted in culture medium $(100 \mu \mathrm{L})$ along the abscissa. A bacterial suspension prepared in fresh culture medium and adjusted to a McFarland standard of 0.5 was used as an inoculum. The microplate wells were inoculated with $100 \mu \mathrm{L}$ of the suspension, and the microplate was incubated at $37{ }^{\circ} \mathrm{C}$ for $24 \mathrm{~h}$ in an anaerobic chamber. Wells with no bacteria or no compounds were included in the assay. After the incubation period, bacterial growth was assessed by recording the $\mathrm{OD}_{660}$ using a Synergy 2 microplate reader. The lowest concentration at which no growth occurred was considered the MIC. The fractional inhibitory concentration index (FICI) was calculated using the following equation: $\mathrm{FICI}=\left(\mathrm{MIC}_{\mathrm{A}+\mathrm{B}} /\right.$ $\left.\mathrm{MIC}_{\mathrm{A}}\right)+\left(\mathrm{MIC}_{\mathrm{B}+\mathrm{A}} / \mathrm{MIC}_{\mathrm{B}}\right)$. An $\mathrm{FICI} \leq 0.5$ was considered as indicating a synergistic effect, an FICI $>0.5$ and $\leq 4.0$ as indicating no effect, and an FICI $>4.0$ as indicating an antagonistic effect. Assays were performed in triplicate to ensure reproducibility. A representative set of data is presented.

\section{Biofilm killing}

The ability of nisin/glabridin, nisin/licoricidin, and nisin/ licochalcone A to kill E. faecalis ATCC 19433 biofilms was investigated. Briefly, 24-h biofilms were pre-formed in the wells of a 96-well microplate, washed once with PBS, and treated for $30 \mathrm{~min}$ with the above combinations. Each compound of a combination was used at a concentration corresponding to its MIC value (in PBS). Following these treatments, the biofilms were washed once with PBS. A series of biofilms was stained with $0.04 \%$ crystal violet as described above to determine biofilm desorption. A second series of biofilms was used to determine bacterial viability using a commercial luminescence assay (BacTiter-Glo ${ }^{\mathrm{TM}}$; Madison, WI, USA) that measures adenosine triphosphate (ATP), an indicator of metabolically active viable bacteria. Luminescence was quantified using a Synergy 2 microplate reader. All the assays were performed in triplicate in two independent experiments, and the means \pm standard deviations were calculated.

\section{In vitro biocompatibility assay}

The human oral epithelial cell line B11, which has already been characterized (Groeger et al. 2008), was kindly provided by S. Groeger (Justus Liebig University Giessen, Germany). Cells were cultured in keratinocyte serum-free medium (K-SFM; Life Technologies Inc., Burlington, ON, Canada) supplemented with growth factors $(50 \mu \mathrm{g} / \mathrm{mL}$ of bovine pituitary extract and $5 \mathrm{ng} /$ $\mathrm{mL}$ of human epidermal growth factor) and $100 \mu \mathrm{g} / \mathrm{mL}$ of penicillin G-streptomycin. The primary human gingival fibroblast cell line HGF-1 (CRL-2014) was purchased from the American Type Culture Collection (ATCC, Manassas, VA, USA) and was cultured in Dulbecco's Modified Eagle's Medium (DMEM; Life Technologies Inc.) supplemented with $4 \mathrm{mM} \mathrm{L}$-glutamine, 15\% heat-inactivated fetal bovine serum (FBS; HyClone Laboratories, Logan, UT, USA), and $100 \mu \mathrm{g} / \mathrm{mL}$ of penicillin G-streptomycin. The previously characterized human SCAP cell line (RP-89) was cultured in -minimum essential medium (MEM; Life Technologies Inc.) supplemented with $10 \% \mathrm{FBS}, 2 \mathrm{mmol} / \mathrm{L}$ L-glutamine, and $100 \mu \mathrm{g} / \mathrm{mL}$ of penicillin G/streptomycin (Ruparel et al. 2013). All cell cultures were incubated at $37^{\circ} \mathrm{C}$ in a $5 \% \mathrm{CO}_{2}$ atmosphere. To evaluate the effect of nisin/ glabridin, nisin/licoricidin, and nisin/licochalcone A on cell viability, cells were seeded $\left(1 \times 10^{5}\right.$ cells in $\left.100 \mu \mathrm{L}\right)$ in the wells of a 96-well tissue culture plate and were incubated at $37{ }^{\circ} \mathrm{C}$ in a $5 \% \mathrm{CO}_{2}$ atmosphere until they reached confluence. The cells were treated with the combinations for $2 \mathrm{~h}$. Each compound of a combination 
was used at concentrations corresponding to the MIC and twofold MIC values obtained against E. faecalis ATCC 19433. Thereafter, an MTT (3-[4, 5-diethylthiazol-2-yl]-2,5-diphenyltetrazolium bromide) assay was performed according to the manufacturer's protocol (Roche Diagnostics, Mannheim, Germany) to assess cell viability. The assays were performed in triplicate in two independent experiments, and the means \pm standard deviations were calculated.

\section{NF-KB activation assay}

The human monoblastic leukemia cell line U937-3хкBLUC was kindly provided by R. Blomhoff (University of Oslo, Norway). It consists of the U937 cell line stably transfected with a luciferase gene coupled to a promoter of three NF- $\mathrm{kB}$ binding sites (Carlsen et al. 2002). Monocytes were grown at $37{ }^{\circ} \mathrm{C}$ in a $5 \% \mathrm{CO}_{2}$ atmosphere in RPMI-1640 medium (Life Technologies Inc.) supplemented with $10 \%$ heat-inactivated FBS, $100 \mu \mathrm{g} / \mathrm{mL}$ of penicillin-streptomycin, and $75 \mu \mathrm{g} / \mathrm{mL}$ of hygromycin B. In a first analysis, the ability of $E$. faecalis ATCC 19433 to induce NF- $\mathrm{kB}$ activation was assessed. Briefly, $100 \mu \mathrm{L}$ of the monocyte suspension $\left(10^{7}\right.$ cells $\left./ \mathrm{mL}\right)$ was seeded in the wells of a black bottom, black wall 96-well microplate (Greiner Bio-One North America Inc., Monroe, NC, USA), and an overnight culture of E. faecalis suspended in culture medium was added at a multiplicity of infection (MOI) of $100,50,25,12,6$, or 3 . The plate was incubated at $37{ }^{\circ} \mathrm{C}\left(5 \% \mathrm{CO}_{2}\right)$ for a further $6 \mathrm{~h}$. NF- $\mathrm{kB}$ activation was then assessed using the Bright-Glo ${ }^{\text {TM }}$ Luciferase Assay System (Promega, Madison, WI, USA) by adding $100 \mu \mathrm{L}$ of luciferase substrate to the wells at room temperature. Luminescence was recorded using the luminometer option of the Synergy 2 microplate reader (BioTek Instruments) within 3 min of adding the substrate. To investigate the ability of nisin/glabridin, nisin/licoricidin, and nisin/licochalcone A (each compound at their MIC, $1 / 2 \mathrm{MIC}$, and $1 / 4$ MIC obtained against E. faecalis ATCC 19433) to inhibit NF- $\mathrm{kB}$ activation, the combinations were added $30 \mathrm{~min}$ prior to challenge U937-3xкB-LUC cells with E. faecalis at an MOI of 100 . All the assays were carried out in triplicate in two independent experiments, and the means \pm standard deviations were calculated.

\section{Statistical analysis}

Statistical analyses were performed using a one-way analysis of variance with a post hoc Bonferroni multiple comparison test (GraphPad Software Inc., La Jolla, CA, USA). All results were considered statistically significant at $p<0.01$.

\section{Results}

The MICs and MBCs of nisin, the licorice polyphenols (glabridin, licoricidin, licochalcone A), and chlorhexidine against three strains of $E$. faecalis are given in Table 1. The MICs of nisin ranged from 12.5 to $25 \mu \mathrm{g} / \mathrm{mL}$. Twice these concentrations of nisin corresponded to the MBCs. The MICs of the licorice polyphenols ranged from 6.25 to $25 \mu \mathrm{g} / \mathrm{mL}$, while the MBCs ranged from 50 to $200 \mu \mathrm{g} / \mathrm{mL}$. In terms of MICs, licoricidin and licochalcone A were slightly more effective than glabridin. Chlorhexidine, which was used as a positive inhibitory control, had MIC and $\mathrm{MBC}$ values of 6.25 and $50 \mu \mathrm{g} / \mathrm{mL}$, respectively.

The effect of a sub-inhibitory concentration (1/4 MIC) of nisin, glabridin, licoricidin, licochalcone $\mathrm{A}$, and chlorhexidine on biofilm formation by $E$. faecalis ATCC 19433 is reported in Table 2. At this concentration, none of the compounds caused a significant decrease in bacterial growth. While glabridin and licochalcone A did not attenuate biofilm formation, nisin and licoricidin caused a reduction in biofilm formation of $31.6 \%$ and $23.5 \%$, respectively. The sub-inhibitory concentration of chlorhexidine reduced the ability of E. faecalis to form a biofilm by $37.5 \%$.

The synergistic antibacterial effect of nisin/glabridin, nisin/licoricidin, and nisin/licochalcone A on E. faecalis ATCC 19433 were assessed using the checkerboard assay. As reported in Table 3, nisin acted in synergy with all three licorice polyphenols. With an FICI of 0.09, nisin/

\begin{tabular}{|c|c|c|c|}
\hline \multirow[t]{2}{*}{ Compound } & \multicolumn{3}{|c|}{ Concentration of compound $(\mu \mathrm{g} / \mathrm{mL})$} \\
\hline & ATCC 19433 & ODOT & 1DOT \\
\hline \multicolumn{4}{|l|}{ Nisin } \\
\hline MIC & 12.5 & 25 & 25 \\
\hline$M B C$ & 25 & 50 & 50 \\
\hline \multicolumn{4}{|l|}{ Glabridin } \\
\hline MIC & 25 & 25 & 25 \\
\hline$M B C$ & 100 & 50 & 50 \\
\hline \multicolumn{4}{|l|}{ Licoricidin } \\
\hline MIC & 6.25 & 12.5 & 6.25 \\
\hline MBC & 50 & 200 & 100 \\
\hline \multicolumn{4}{|c|}{ Licochalcone A } \\
\hline MIC & 12.5 & 12.5 & 12.5 \\
\hline $\mathrm{MBC}$ & 100 & 200 & 200 \\
\hline \multicolumn{4}{|l|}{ Chlorhexidine } \\
\hline MIC & 6.25 & 6.25 & 6.25 \\
\hline $\mathrm{MBC}$ & 50 & 50 & 50 \\
\hline
\end{tabular}


Table 2 Effects of nisin, licorice polyphenols (glabridin, licoricidin, licochalcone A), and chlorhexidine at a subinhibitory concentration (1/4 MIC) on biofilm formation by E. faecalis ATCC 19433

\begin{tabular}{llc}
\hline Compound $(\boldsymbol{\mu g} / \mathbf{m L})$ & Relative growth (\%) & Biofilm formation (\%) \\
\hline None & $102.3 \pm 2.5$ & $101.5 \pm 4.9$ \\
Nisin $(3.125 \mu \mathrm{g} / \mathrm{mL})$ & $109.1 \pm 9.9$ & $76.5 \pm 6.6^{*}$ \\
Glabridin $(6.25 \mu \mathrm{g} / \mathrm{mL})$ & $101.4 \pm 2.9$ & $103.8 \pm 6.8$ \\
Licoricidin $(1.56 \mu \mathrm{g} / \mathrm{mL})$ & $104.4 \pm 2.2$ & $68.4 \pm 4.9^{*}$ \\
Licochalcone A & $97.1 \pm 0.9$ & $91.4 \pm 8.7$ \\
$\quad(3.125 \mu \mathrm{g} / \mathrm{mL})$ & \\
$\begin{array}{c}\text { Chlorhexidine }(1.56 \mu \mathrm{g} / \\
\mathrm{mL})\end{array}$ & $106.8 \pm 3.7$ & $62.5 \pm 1.5^{*}$ \\
\hline
\end{tabular}

*Significant reduction $(p<0.01)$ compared to control cultures without compounds

Table 3 Fractional inhibitory concentration index (FICI) values for combinations of nisin and licorice polyphenols against E. faecalis ATCC 19433

\begin{tabular}{llll}
\hline Compound A & Compound B & FICl* $^{*}$ & Effect \\
\hline Nisin & Glabridin & 0.09 & Synergistic \\
Nisin & Licoricidin & 0.25 & Synergistic \\
Nisin & Licochalcone A & 0.5 & Synergistic \\
\hline
\end{tabular}

${ }^{*} \mathrm{FICl} \leq 0.5$ : synergistic effect; $\mathrm{FICl}>0.5$ and $\leq 4.0$ : no effect; $\mathrm{FICl}>4.0$ : antagonistic effect

Table 4 Effects of nisin/glabridin, nisin/licoricidin, and nisin/licochalcone $A$ on the killing of a pre-formed $E$. faecalis ATCC 19433 biofilm after a 30-min contact

\begin{tabular}{ll}
\hline Compound & \% biofilm killing \\
\hline None (PBS treatment) & 0 \\
Nisin $(12.5 \mu \mathrm{g} / \mathrm{mL})+$ Glabridin $(25 \mu \mathrm{g} / \mathrm{mL})$ & $61.4 \pm 6.0^{*}$ \\
Nisin $(12.5 \mu \mathrm{g} / \mathrm{mL})+$ Licoricidin $(6.25 \mu \mathrm{g} / \mathrm{mL})$ & $75.8 \pm 3.1^{*}$ \\
Nisin $(12.5 \mu \mathrm{g} / \mathrm{mL})+$ Licochalcone $A(12.5 \mu \mathrm{g} / \mathrm{mL})$ & $76.4 \pm 3.0^{*}$ \\
\hline
\end{tabular}

*Significantly different $(p<0.01)$ from control biofilms treated with PBS

Table 5 Effects of nisin/glabridin, nisin/licoricidin, and nisin/licochalcone $A$ on the viability of oral epithelial cells, gingival fibroblasts, and stem cells of the apical papilla (SCAP) after a 2-h contact

\begin{tabular}{|c|c|c|c|}
\hline \multirow[t]{2}{*}{ Mixtures } & \multicolumn{3}{|l|}{ Cell viability (\%) } \\
\hline & Oral epithelial cells & Gingival fibroblasts & SCAP \\
\hline None (control) & 100 & 100 & $111.8 \pm 9.5$ \\
\hline Nisin $(25 \mu \mathrm{g} / \mathrm{mL}) /$ Glabridin $(50 \mu \mathrm{g} / \mathrm{mL})$ & $71.5 \pm 9.2^{*}$ & $82.5 \pm 5^{*}$ & $5.0 \pm 2.1^{*}$ \\
\hline Nisin $(12.5 \mu \mathrm{g} / \mathrm{mL}) /$ Glabridin $(25 \mu \mathrm{g} / \mathrm{mL})$ & $115.0 \pm 19.4$ & $148.2 \pm 11.0$ & $122.4 \pm 3.6$ \\
\hline Nisin $(25 \mu \mathrm{g} / \mathrm{mL}) / \mathrm{Licoricidin}(12.5 \mu \mathrm{g} / \mathrm{mL})$ & $134.3 \pm 2.4$ & $116.0 \pm 2.5$ & $107.2 \pm 6.0$ \\
\hline Nisin $(12.5 \mu \mathrm{g} / \mathrm{mL}) / \mathrm{Licoricidin}(6.25 \mu \mathrm{g} / \mathrm{mL})$ & $131.1 \pm 3.6$ & $113.5 \pm 5.4$ & $104.2 \pm 8.4$ \\
\hline Nisin $(25 \mu \mathrm{g} / \mathrm{mL}) /$ Licochalcone A (25 $\mu \mathrm{g} / \mathrm{mL})$ & $139.3 \pm 4.2$ & $99.8 \pm 14.1$ & $116.9 \pm 6.7$ \\
\hline  & $137.0 \pm 5.6$ & $104.0 \pm 18.3$ & $114.4 \pm 4.5$ \\
\hline
\end{tabular}

glabridin displayed the most marked synergistic antibacterial effect.

Given the synergistic antibacterial interactions observed with the combinations of nisin and the licorice polyphenols, the second part of the present study investigated nisin/glabridin, nisin/licoricidin, and nisin/licochalcone $\mathrm{A}$, which were tested for their bactericidal effects on pre-formed E. faecalis biofilms. Following a $30 \mathrm{~min}$ contact, none of the combinations caused the desorption of the biofilm (data not shown). As reported in Table 4, nisin/licoricidin and nisin/licochalcone $\mathrm{A}$ resulted in $>75 \%$ biofilm killing, while nisin/glabridin resulted in $61.4 \%$ killing.

As the biocompatibility of antimicrobial agents used for root canal disinfection is an important aspect that must be taken into consideration, we investigated the cytotoxic effects of nisin/glabridin, nisin/licoricidin, and nisin/licochalcone A on three human cell types: oral epithelial cells, gingival fibroblasts, and stem cells of the apical papilla. As reported in Table 5, with the exception of nisin/glabridin (both compounds used at their MIC) that caused a reduction in the viability stem cells of the apical papilla, all the combinations tested exhibited low toxicity.

Lastly, we investigated whether nisin/glabridin, nisin/ licoricidin, and nisin/licochalcone A exert an anti-inflammatory effect through the inhibition of NF- $\mathrm{kB}$ activation using a monocyte cell line stably transfected with a luciferase gene coupled to a promoter of three NF- $\mathrm{\kappa B}$ binding sites. We first showed that $E$. faecalis activates the NF- $\mathrm{BB}$ signaling pathway in a dose-dependent manner (Fig. 1). We observed a 7.5-fold activation of the NF- $\mathrm{kB}$ signaling pathway at an MOI of 50 . The monocytes were preincubated for $30 \mathrm{~min}$ with the mixtures containing nisin and the licorice polyphenols at their MICs, $1 / 2 \mathrm{MICs}$, and $1 / 4$ MICs prior to stimulating the cells with E. faecalis at an MOI of 50. As shown in Fig. 2, all the combinations dose-dependently inhibited E. faecalis-mediated NF-кB activation. More specifically, when used at a concentration corresponding to $1 / 4 \mathrm{MIC}$, nisin/glabridin, nisin/

*Significant decrease $(p<0.01)$ compared to untreated control cell 


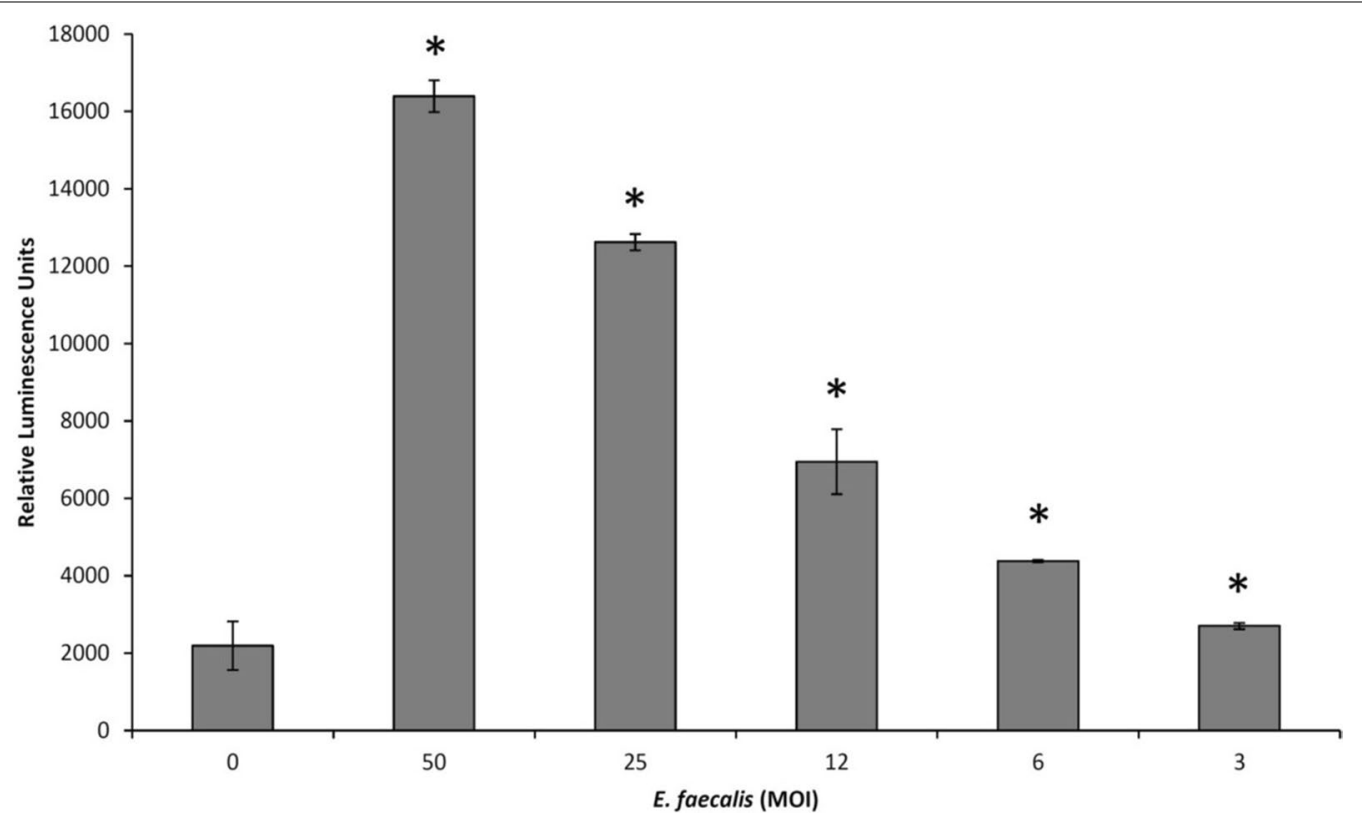

Fig. 1 Effects of E. faecalis ATCC 19433 on NF-KB activation using the human monoblastic leukemia cell line U937-3xkB-LUC. *Significant increase $(p<0.01)$ compared to unstimulated control cells

licoricidin, and nisin/licochalcone A reduced NF-kB activation by $38.9 \%, 66.4 \%$, and $36.9 \%$, respectively. No cytotoxic effects were associated with these treatments (data not shown).

\section{Discussion}

E. faecalis is the bacterial species most commonly associated with failed root canal treatments and persistent root canal infections (Roças et al. 2004; Sedgley et al. 2006). This is related to its ability to colonize the dentinal tubules, form a biofilm, survive nutrient limitation, and be particularly resistant to several antimicrobial agents. E. faecalis resistant to root canal treatments can sustain an inflammatory response leading to chronic apical periodontitis. Although a number of intracanal medications have been proposed for the chemo-mechanical preparation of the root canal, the search for novel alternatives is still an active field of research. The aim of the present study was to evaluate the synergistic antibacterial effects of nisin and selected licorice polyphenols (glabridin, licoricidin, licochalcone A) on planktonic and biofilmembedded $E$. faecalis cells. The biocompatibility and anti-inflammatory properties of nisin/licorice polyphenol combinations were also investigated.

Nisin A, a lantibiotic bacteriocin produced by L. lactis that mainly exhibits antibacterial activity against Grampositive bacteria, is particularly relevant with regard to persistent endodontic infections involving $E$. faecalis. We showed that this antimicrobial peptide was active against E. faecalis, with MBCs ranging from 12.5 to $25 \mu \mathrm{g} / \mathrm{mL}$. This in agreement with a previous study by Kajwadkar et al. (2017), who reported that nisin Z, which differs from nisin A by a single amino acid substitution at position 27, has antibacterial activity against E. faecalis. Interestingly, nisin has also been reported to be active against a number of oral pathogens associated with dental caries and periodontal diseases (Shin et al. 2015; Tong et al. 2014). We then showed that two isoflavonoids (glabridin, licoricidin) and a chalcone (licochalcone $\mathrm{A}$ ), which are important constituents of licorice root, also efficiently inhibit the growth of E. faecalis, with MICs ranging from 6.25 to $25 \mu \mathrm{g} / \mathrm{mL}$.

We used a broth microdilution checkerboard assay to show that combinations of nisin and licorice polyphenols exhibited significant synergistic antibacterial effects, especially nisin/glabridin. Although the exact mechanism of synergy is not understood, it may be related to the fact that nisin and the licorice polyphenols exert their antibacterial effect via different mechanisms. For instance, the membrane-permeabilizing activity of nisin (Lubelski et al. 2008) may increase the uptake of licorice polyphenols that, in turn, may have an intracellular target. Interestingly, the combinations of nisin and licorice polyphenols were also effective in the killing of pre-formed biofilms of E. faecalis. Further studies should evaluate theirs effects on polymicrobial biofilms, as observed in infected root canals. 


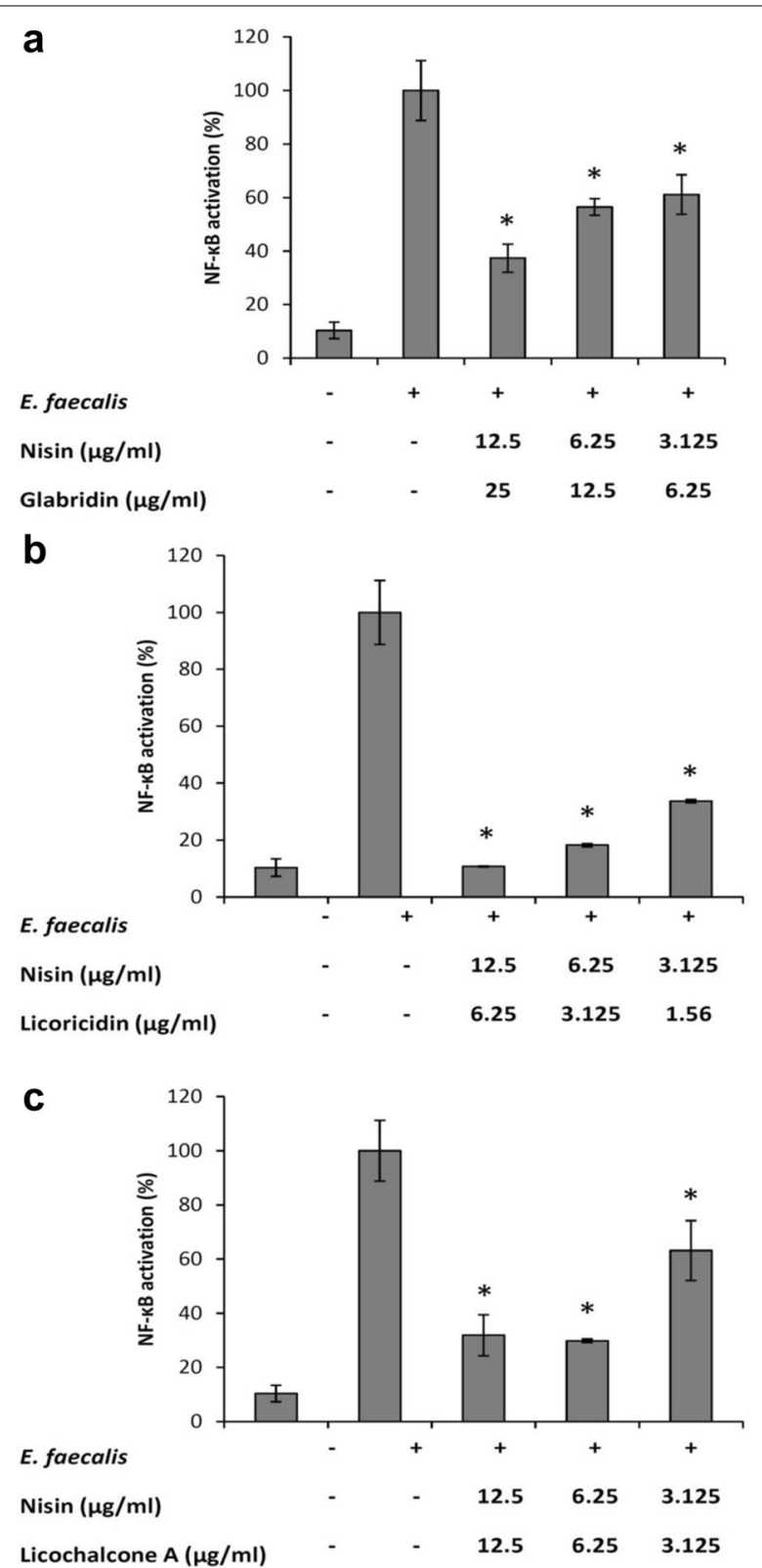

Fig. 2 Effects of nisin/glabridin, nisin/licoricidin, and nisin/ licochalcone A on E. faecalis-mediated NF-KB activation using the human monoblastic leukemia cell line U937-3×KB-LUC. *Significant reduction $(p<0.01)$ compared to E. faecalis-stimulated cells (no compounds)

Nisin is well known to be mostly active against Grampositive bacteria (Lubelski et al. 2008), while root canal infections also involve Gram-negative bacteria. Combining this bacteriocin with licorice polyphenols allows to develop an irrigating solution with a much larger spectrum of antimicrobial activity. Indeed, Marcoux et al.
(2020) recently reported that licochalcone A, licoricidin, and glabridin had MIC values ranging from 1.56 to $50 \mu \mathrm{g} /$ $\mathrm{mL}$ against Gram-negative endodontic bacterial pathogens as well as Candida albicans.

In view of their potential use for root canal disinfection, determining the biocompatibility of the active compounds is critical. We used three different cell models (oral epithelial cells, gingival fibroblasts, and stem cells of the apical papilla) to show that the nisin/licorice polyphenol combinations had no cytotoxic effects, with the exception of nisin/glabridin, when used at their MICs. This is in agreement with a previous study by Shin et al. (2015), who reported that orally relevant human cells, including oral keratinocytes, gingival fibroblasts, periodontal ligament cells, and osteoblast-like cells, are not affected by up to $200 \mu \mathrm{g} / \mathrm{mL}$ of nisin.

Endodontic pathogens and the toxins they release can affect the periradicular tissues and induce periapical inflammatory lesions, which are characterized by the recruitment of inflammatory cells, including polymorphonuclear neutrophils and macrophages (Gomes and Herrera 2018). The transcription factor NF-kB of inflammatory cells is activated by a wide variety of stimuli, including bacterial pathogens, and has many target genes, including those that encode cytokines and matrix metalloproteinases that modulate periradicular tissue and bone destruction (Kumar et al. 2004). NF- $\mathrm{kB}$ is thus a central player in inflammatory diseases such as apical periodontitis, and the inhibition of its activation is considered a promising therapeutic strategy (Gupta et al. 2010). In the present study, we showed that nisin/glabridin, nisin/licoricidin, and nisin/licochalcone A inhibit NF- $\kappa B$ activation induced by E. faecalis in a monocyte model, suggesting that these combinations possess antiinflammatory properties. This supports a previous study by Bodet et al. (2008), who investigated the effect of a licorice extract on the periodontopathogen-induced inflammatory response in macrophage and whole blood models and found that the extract exerted a potent antiinflammatory effect by inhibiting the secretion of several pro-inflammatory cytokines, including IL-1 $\beta$, IL-6, IL-8, and TNF- $\alpha$. Moreover, licoricidin reduced inflammatory cytokine and matrix metalloproteinase secretion in a macrophage model stimulated with LPS (La et al. 2011), while licochalcone A has been reported to inhibit NF- $\mathrm{kB}$ activation (Furusawa et al. 2009).

In conclusion, the present study showed that combinations of nisin and glabridin, licoricidin, or licochalcone possess a number of desirable properties for root canal irrigation such as antibacterial activity, anti-inflammatory activity, and lack of cytotoxicity. Future studies should thus focus on determining whether these combinations can dissolve organic matter. Although our in vitro results 
were promising, clinical studies will be needed to assess their efficacy and safety.

\section{Acknowledgements}

We thank S. Groeger and J. Meyle (Justus-Liebig-University Giessen, Germany) for kindly providing the B11 keratinocyte cell line. We thank A. Diogenes (University of Texas Health Science Center at San Antonio, USA) for providing the RP-89 cell line. We are also grateful to R. Blomhoff and H. Carlsen (University of Oslo, Norway) for providing the U937-3xKB-LUC cell line and to Brenda Gomes (State University of Campinas) for supplying clinical strains of E. faecalis. The technical assistance of G. LeBel and K. Vaillancourt is acknowledged.

\section{Authors' contributions}

$E M, J A$ and $A B L$ contributed to the acquisition, analysis, and interpretation of data. PG and DG contributed to the conception and design of the study. All authors were involved in drafting and critically revising the manuscript, and approved the final version submitted to the journal. All authors read and approved the final manuscript.

\section{Funding}

This study was financially supported by Réseau de Recherche en Santé Buccodentaire et Osseuse of the Fonds de Recherche du Québec-Santé, the AAE Foundation of Endodontics, and the Canadian Academy of Endodontics Endowment Fund.

\section{Availability of data and materials}

The corresponding author is responsible for providing all experimental data upon request.

\section{Ethics approval and consent to participate}

Not applicable.

\section{Consent for publication}

Not applicable.

\section{Competing interests}

The authors declare that they have no competing interests.

Received: 15 May 2020 Accepted: 26 June 2020

Published online: 06 July 2020

\section{References}

Bodet C, La VD, Gafner S, Bergeron C, Grenier D (2008) A licorice extract reduces lipopolysaccharide-induced proinflammatory cytokine secretion by macrophages and whole blood. J Periodontol 79:1752-1761

Carlsen H, Moskaug JO, Fromm SH, Blomhoff R (2002) In vivo imaging of NFkappa B activity. J Immunol 168:1441-1446

Daglia M (2012) Polyphenols as antimicrobial agents. Curr Opin Biotechnol 23:174-181

De Sermeno F, da Silva LA, Herrera H, Herrera H, Silva RAB, Leonardo MR (2009) Tissue damage after sodium hypochlorite extrusion during root canal treatment. Oral Surg Oral Med Oral Pathol Oral Radiol Endod 108:E46-E49

Duggan JM, Sedgley CM (2007) Biofilm formation of oral and endodontic Enterococcus faecalis. J Endod 3:815-818

Eliopoulos GM, Moellering RC (1996) Antimicrobial combinations. In: Lorian V (ed) Antibiotics in laboratory medicine. Lippincott Williams and Wilkins, Baltimore, pp 330-396

Fraga CG, Croft KD, Kennedy DO, Tomas-Barberan FA (2019) The effects of polyphenols and other bioactives on human health. Food Funct 10:514-528

Furusawa JI, Funakoshi-Tago M, Mashino T, Tago K, Inoue H, Sonoda Y, Kasahara T (2009) Glycyrrhiza inflate-derived chalcones, licochalcone A, licochalcone B and licochalcone D, inhibit phosphorylation of NF-kB p65 in LPS signaling pathway. Int Immunopharmacol 9:499-507

Gomes BPFD, Herrera DR (2018) Etiologic role of root canal infection in apical periodontitis and its relationship with clinical symptomatology. Braz Oral Res 32:e69

Goncalves LS, Rodrigues RCV, Andrade CV, Soares RG, Vettore MV (2016) The effect of sodium hypochlorite and chlorhexidine as irrigant solutions for root canal disinfection: a systematic review of clinical trials. J Endod 42:527-532

Groeger S, Michel J, Meyle J (2008) Establishment and characterization of immortalized human gingival keratinocyte cell lines. J Periodontal Res 43:604-614

Gupta SC, Sundaram C, Reuter S, Aggarwal BB (2010) Inhibiting NF-kB activation by small molecules as a therapeutic strategy. Biochim Biophys Acta 1799:775-787

Hansen JN (1994) Nisin as a model food preservative. Crit Rev Food Sci Nutr 34:69-93

Kajwadkar R, Shin JM, Lin GH, Fenno JC, Rickard AH, Kapila YL (2017) Highpurity nisin alone or in combination with sodium hypochlorite is effective against planktonic and biofilm populations of Enterococcus faecalis. J Endod 43:989-994

Kumar A, Takada Y, Boriek AM, Aggarwal BB (2004) Nuclear factor-kappaB: its role in health and disease. J Mol Med 82:434-448

La VD, Tanabe S, Bergeron C, Gafner S, Grenier D (2011) Modulation of matrix metalloproteinase and cytokine production by licorice isolates licoricidin and licorisoflavan A: potential therapeutic approach for periodontitis. J Periodontol 82:122-128

Lubelski J, Rink R, Khusainov R, Moll GN, Kuipers OP (2008) Biosynthesis, immunity, regulation, mode of action and engineering of the model lantibiotic nisin. Cell Mol Life Sci 65:455-476

Marcoux E, Ben Lagha A, Gauthier P, Grenier D (2020) Antimicrobial activities of natural plant compounds against endodontic pathogens and biocompatibility with human gingival fibroblasts. Arch Oral Biol 116:104734

Messier C, Epifano F, Genovese S, Grenier D (2012) Licorice and its potential beneficial effects in common oro-dental diseases. Oral Dis 18:32-39

Roças IN, Siqueira JF Jr, Santos KR (2004) Association of Enterococcus faecalis with different forms of periradicular diseases. J Endod 30:315-320

Ruparel NB, de Almeida JF, Henry MA, Diogenes A (2013) Characterization of a stem cell of apical papilla cell line: effect of passage on cellular phenotype. J Endod 39:357-363

Sedgley C, Nagel A, Dahlen G, Reit C, Molander A (2006) Real-time quantitative polymerase chain reaction and culture analysis of Enterococcus faecalis in root canals. J Endod 32:173-177

Shin JM, Ateia I, Paulus JR, Liu H, Fenno JC, Rickard AH, Kapila YL (2015) Antimicrobial nisin acts against saliva derived multi-species biofilms without cytotoxicity to human oral cells. Front Microbiol 6:617

Siqueira JF, Rocas IN (2009) Diversity of endodontic microbiota revisited. J Dent Res 88:969-981

Tong Z, Ni L, Ling J (2014) Antibacterial peptide nisin: a potential role in the inhibition of oral pathogenic bacteria. Peptides 60:32-40

Vidana R, Sullivan A, Billstrom H, Ahlquist M, Lund B (2011) Enterococcus faecalis infection in root canals-host-derived or exogenous source? Lett Appl Microbiol 2:109-115

Wayne PA (2012) Methods for dilution antimicrobial susceptibility tests for bacteria that grow aerobically; approved standard, Ninth Edition. CLSI document M07-A9. Clinical and Laboratory Standards Institute

\section{Publisher's Note}

Springer Nature remains neutral with regard to jurisdictional claims in published maps and institutional affiliations. 